\title{
Foreword of the Founding Editor of the Journal Transport in Porous Media, Jacob Bear
}

\author{
Jacob Bear ${ }^{1}$ \\ Published online: 16 August 2021 \\ (c) The Author(s), under exclusive licence to Springer Nature B.V. 2021
}

I'll start by expressing my most sincere thanks to Martin Blunt, Majid Hassanizadeh, and Brian Berkowitz, for initiating and implementing the idea of a special issue of TIPM to honor my initiative some 37 years ago, of starting a new Springer scientific/professional journal devoted to phenomena of transport of extensive quantities in porous medium domains. Indeed, this is a great honor for me. THANKS!

Let me tell you about myself. I was born (1929) in Israel (then British mandate Palestine). My parents immigrated to Israel as Zionists driven by the urge to participate in the establishment of a homeland for the Jews in their ancestral land so that Jews will have at least one place in the world to where they can flee whenever persecuted. I married my wife, Siona, in 1951. We have three children and six grandchildren. Finishing high school (1946), I joined the PALMACH (the Israeli underground fighting units) and fought in the War of Independence (1947-8).

In 1949, I started my studies of Civil Engineering at the Technion-Israel Institute of Technology, Haifa. I elected to graduate in the option of water resources, realizing that water would be a central issue and a limiting factor in Israel's development. I liked the challenge. I received my B.Sc. in 1953 and started to work as an engineer in the Planning Division of TAHAL-Water Planning for Israel Ltd., the government (now private) company in charge of water resources planning and development in Israel. Awarded a scholarship by the Dutch government, I spent 1955/6 at the Government Institute for Water Supply (Scheveningen, NL), conducting research on artificial recharge of groundwater and on seawater intrusion. I used laboratory models, especially the Hele-Shaw (parallel-plate) model, as tools for solving ground water flow and storage problems. During the 1960s (no computers, but practical problems had to be solved!), I used these (horizontal and vertical) models extensively to investigate seawater intrusion into layered coastal aquifers under the sharp interface approximation (my Technion M.Sc., 1957), and artificial recharge of aquifers.

Returning from the Netherlands, I moved to the groundwater department of TAHAL. In Israel, at that time, groundwater, primarily from the coastal (sandstone) aquifer and the (limestone) mountain aquifer, constituted the major source of water. However, the total available annual sustainable water yield is rather small, to the extent that it seriously constrains development. Seawater intrusion was high on my agenda. I worked under the

Jacob Bear

cvrbear@technion.ac.il

1 Department of Civil and Environmental Engineering, Technion, Israel Institute of Technology, Haifa 32000, Israel 
simplified assumption of a sharp interface, However, field measurements showed that the approximation of a "sharp interface" was not justified. A literature survey indicated that the same problem, of seawater intrusion, was also encountered and investigated in California. A scholarship brought me to the University of California at Berkeley in 1958. There, I spent the next two years under the supervision of Professor David K. Todd, investigating seawater intrusion into coastal aquifers without the assumption of a sharp interface. I investigated the spreading of a solute, referred to as "dispersion". At that time, I could already find some articles on dispersion, or "miscible displacement", as it was called then. I would like to mention the works of P.G. Gerard de Josselin de Jong (1958) of TUDelft, Saffman (1959) in the UK, and Scheidegger. As part of my Ph.D. research, assuming a linear relationship to velocity in homogeneous domains, I developed the concept of dispersion coefficient as a second rank tensor and the dispersivity as a fourth rank tensor. These results (Bear 1961) formed the basis for understanding mechanical dispersion and the role of diffusion.

Returning from Berkeley in 1960, I joined the Department of Civil Engineering at the Technion-Israel Institute of Technology, teaching and conducting research in engineering hydrology, groundwater hydrology, and seawater intrusion into coastal aquifers, developing a comprehensive theory of (mathematical) modeling phenomena of transport (of mass, momentum, and energy) in porous medium domains. I was promoted to the rank of professor in 1970. Over the years, I served in a number of administrative positions at Technion, including Vice President for Academic Affairs (1970-1976), first director of the Neaman Institute for Advanced Studies in Science and Technology (1978-1981), Dean of the Graduate School (1983-1986), and Dean of Civil Engineering (1995-1997).

During 1960-1989, in parallel to my academic activities at Technion, I served as an active consultant on Groundwater Hydrology and the Development of Water Resources to TAHAL and to Israel's Water Commissioner. I was deeply involved in the development of water resources in Israel, as well as in educating engineers and hydrologists in using scientific modeling approaches in their practice.

As I continued to work on ground water flow, drainage, contamination of aquifers, modeling migration of fluids in oil reservoirs, agricultural drainage, and similar subjects, using books like Muskat (1937, 1946), Aravin and Numrov (1953), Collins (1961), Luthin (1966) Scheidegger (1960), and many scientific and professional papers in professional journals, I realized that, although they belong to different scientific/engineering professions and speak different "languages," all these authors are actually dealing with applications of a single fundamental theory that deals with phenomena of transport (movement, storage, and transformation) of extensive quantities (such as mass, mass of chemical components, momentum, and energy) in the special domains referred to as "porous medium." I started to develop, write and lecture on this unified approach.

In 1980, I was appointed to the Albert and Anne Mansfield Chair in Water Resources at Technion. In 1988, I was awarded an honorary doctorate in technological sciences by E.T.H. Zurich, Switzerland, and was elected Fellow of the American Geophysical Union. In 1998, I was awarded the Rothschild Prize in Engineering in Israel. In 2003, I was awarded the Excellence in Geophysical Education Medal by the American Geophysical Union.

I have always enjoyed teaching and educating (not a sin, I hope, for a professor) the new generation of hydrologists. I had the opportunity to teach at many universities during sabbaticals. I have had excellent Ph.D. students. I have also offered (and still do) many short courses for professionals (hydrologists and modelers) at many universities and institutes around the world, presenting the mathematical models of flow and 
contaminant transport in the subsurface, and emphasizing the importance of selecting the correct conceptual models. In recent years, I have become enthusiastic about distance learning through the Internet as an important educational tool for students and active professionals.

In 1967, I established a Section of Flow Through Porous Media within the framework of the International Association for Hydraulic Research, and served as its chairman for four years. In 1969, I convened at the Technion, the First International Symposium on the Fundamentals of Transport Phenomena in Porous Media. It brought together professionals and scientists from many disciplines, all working in and making use of the same fundamental theory. A second conference with the same objectives was convened a few years later in Guelph, Canada.

These ideas brought me to write the book Dynamics of Fluids in Porous Media (Bear 1972; also available in Chinese). In it, I presented a unified approach and a theory that is applicable to many professional disciplines, starting from the concept of an REV and the definition of a porous medium. The emphasis in this book was not only on the description of phenomena, but also on the use of well-posed mathematical models as a tool for describing phenomena of transport in porous media, and for forecasting the behavior of fluid and solid phases in response to excitations. The computers and computer programs provided the solutions that made such models practical tools and not merely scientific exercises.

The ideas of a unified fundamental theory underlying the verbal description and mathematical modeling of phenomena of transport in porous media, with applications in many disciplines, led me in 1985 to establish the SPRINGER journal Transport in Porous Media (TIPM), aimed at bringing scientists and professionals from many disciplines under the same roof. I have served as its editor for 25 years, passing the role to Prof. Martin Blunt who is doing excellent work in making TIPM an excellent journal.

And to complete the story of making transport in porous media a full-fledged scientific discipline, Majid Hassanizadeh and colleagues have established (2008) the most successful International Society for Porous Media, InterPore, which assembles under the same scientific-professional roof scientists and industries engaged in a wide spectrum of research and practice in this (very wide!) field.

However, and although I have recently (2018) published a Springer book with the title Modeling Phenomena of Flow and Transport in Porous Media (actually, I could have omitted "Flow and"), I wish to share with you some thoughts on the subject. Aren't we all-those who deal with the general theory of modeling the transport in porous media-really dealing with nothing more than just continuum mechanics (and thermodynamics) of a solid-fluid mixture called "porous medium?" In the (classical) continuum mechanics, we consider the motion and change of storage of the extensive quantities mass, momentum, and energy at a point in a considered spatial phase domain assumed to behave as a continuum, i.e., phase values can be assigned to, and monitored, at every point in that domain. We do so by defining a microREV centered at every point within the considered phase domain and (conceptually) assigning to that center average properties of the molecules within that volume. In this way, fluid and solid continua are created. This is the foundation of Continuum Mechanics as applied to a single phase and can easily be applied to two or more fluid and solid phases in a domain with a known sharp interface between adjacent phases. By averaging over the microREV and assigning the result to a point, we lose the information concerning the molecular behavior in the vicinity of that point. We compensate for this loss by introducing phase properties: density, viscosity, diffusivity, thermal conductivity, etc., assigned to phase points. 
In a porous medium domain, we use the concept of an REV to represent the averaged behavior of solid and fluid phases in the vicinity of a point, assigning this behavior to the considered point. Again, we obtain the behavior of a continuum for every considered phase present at/in the close vicinity of a point, with the possibility of interaction between the phases at a point. Thus, the continuum approach, initially introduced for passing from the molecular level to the microscopic (continuum) one, has been extended to describe phenomena of transport in porous medium domains at the macroscopic-level continuum. In this approach, the missing information concerning interphase boundaries and their effects on phenomena of transport of extensive quantities are replaced by coefficients such as porosity, specific interphase area, permeability, dispersivity. Altogether, transport in porous media is continuum mechanics and thermodynamics in porous medium domains.

In 1979, I published Hydraulics of Groundwater, in which I tried to present a comprehensive approach and mathematical modeling of flow and contaminant transport in groundwater hydrology. I wrote this book because I was convinced that mathematical models were a tool that should be available to and used by ground water consultants and professionals, and not only by scientists and special experts. The book contained a chapter on flow in the unsaturated zone (usually treated by soil physicists), which I realized was going to play an important role in modeling subsurface contamination originating at ground surface, and a chapter on ground water management in which the flow and transport models serve as constraints.

I coauthored Modeling Groundwater Flow and Pollution (Bear and Verruijt 1987), combining the same approach with the presentation of a simple computer code (by Arnold Verruijt). I also coauthored Introduction to Modeling of Transport Phenomena Porous Media (Bear and Bachmat 1990), a comprehensive, unified approach to modeling phenomena of transport in porous media, from the microscopic to the averaged continuum level. Nowadays, mathematical models are accepted as fundamental tools in practice, but not long ago the question of whether models should legitimately be used as a prediction tool was still being debated (e.g., the International Ground Water Modeling Center was established in 1987 to promote the use of models. For some years, I served on its International Technical Advisory Committee.)

Over the years, I observed how the emphasis in ground water investigations (in both research and practice) has been shifting from flow in aquifers, including evaluation of aquifer yield, estimating aquifer properties, and hydraulics of wells, to the current emphasis on contamination (and remediation) of the subsurface. In the 90's, I had the chance to work on these subjects, in connection with cleanup operations, with a wonderful, experienced, and knowledgeable team at Lawrence Livermore National Laboratory (LLNL) and Weiss Associates, Emeryville, CA.

During the period 2002-2017, I have been engaged in a number of research projects sponsored by the European Community. eg., on the prevention of seawater intrusion, on artificial recharge of aquifers and on $\mathrm{CO}_{2}$ sequenstration in deep geological formations, in an effort to mitigate climate change, conducted within the framework of the European Community. All projects were conducted as a cooperation of a number of research organizations and universities in Europe.

This is it. For me, working in the area of groundwater, and in the general field of modeling phenomena of transport in porous media, has been and still is exciting and rewarding. It is a field that requires teamwork - the cooperation of geologists, hydrologists, chemical engineers, biologists, and computer experts, to mention but a few. It is 
certainly a field in which one can contribute significantly to society and to the quality of our environment. I have tried to contribute to this exciting field.

Again thanks to all those who have contributed to this TIPM issue,

Prof. (Emeritus) Jacob Bear

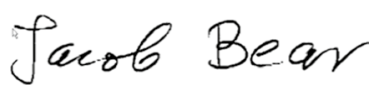

Publisher's Note Springer Nature remains neutral with regard to jurisdictional claims in published maps and institutional affiliations. 